\title{
Numerical investigation of multiphase supersonic swirl flow inside convergent-divergent nozzle
}

The Journal of Computational

\author{
Ehsan Eslamian and Hassan Shirvani
}

\begin{abstract}
Numerical study of multiphase swirl flow induced by helical insert inside the supersonic nozzle is investigated as applied in sandblasting systems. The finding of this research is crucial in improving the performance of abrasive blasting systems by reducing operation time and saving energy. Simulations are performed with Reynolds Stress Model turbulence modelling to capture anisotropy inside the flow. To simulate particles inside the nozzle, discrete phase model has been applied. The multiphase Eulerian method did not provide accurate results on modelling particles with small volume fraction $(<10 \%)$, and also it was not capable of modelling particles interaction. The analyses are performed at three inlet pressures (2, 3 and $4 \mathrm{~atm})$ with constant mass flow rate for particles. The results show that swirl effect increases the mixing feature of the flow, and therefore increases the cleaning area on a working surface. Furthermore, with swirl there was less reduction in maximum velocity compare with single phase simulations, hence particles will have greater speed at the exit of the nozzle.
\end{abstract}

\section{Keywords}

Computational Fluid Dynamics (CFD), swirl, discrete phase model, multiphase, supersonic, converging-diverging nozzle

Date received: I8 November 2015; accepted: II February 2016

\section{Introduction}

Investigation of supersonic flow inside convergingdiverging nozzles has been the subject of several numerical and experimental studies in the past ${ }^{1,2}$ because of their great application in propulsion, steam turbines and sandblasting, but there is not much research on the effect of swirl flow inside converging-diverging nozzles. Swirling flows are very common in technical applications, such as turbo machinery, cyclone or separators, and they require rather sophisticated modelling. Effect of swirl inside nozzle can intensify heat and mass transfer and improve mixing features of the flow by increasing turbulence and vorticity, which can be useful not only in sandblasting applications, but also in combustion injectors for flame stability $^{3}$ or jet engines for noise reduction, ${ }^{4,5}$ and changing the shock structure and its interaction with boundary layer. These several viscous and compressible phenomena affect the flow behaviour inside and outside of the nozzle.

The need for accurate understanding of flow features inside nozzle with swirl is important to predict its effect. Since experimental data for the swirl flow inside highspeed nozzle are scare; the numerical results are vital for understanding the flow and further analyses. This study is based on standard sandblasting nozzle, where single phase simulations on it with helical insert was investigated by Eslamian et al. ${ }^{6}$

\section{Sandblasting}

Sandblasting, which is formed from nozzle with a mixture of air, water and abrasive media, has been used for many industrial applications such as cleaning or removing coating from different type of surfaces, surface strengthening and surface modification ${ }^{7}$ and can provide perfect surface treatment for different materials like plastics, composites, steel, etc. ${ }^{8}$ In sandblasting treatment, abrasive material is accelerated through a nozzle due to the pressure difference. As a Department of Engineering and Built Environment, Anglia Ruskin
University, UK

\section{Corresponding author:}

Ehsan Eslamian, Department of Engineering and Built Environment, Anglia Ruskin University, Chelmsford CMI ISQ, UK.

Email: ehsan.eslamian-koupaei@student.anglia.ac.uk 
result, in any sandblasting method nozzle geometry, jet velocity and impact angle ${ }^{9}$ are the most important parameters to improve effectiveness of sandblasting system. There are different studies and patents to improve sandblasting and make the process faster and more efficient, but none of them are working on fluid dynamic and pressure distribution inside nozzle.

Sandblasting machines operate at a pressure range from 30 to 130 psi. However, for the most applications they are operating at pressures less than $60 \mathrm{psi}$ to avoid damaging a working surface and to save energy. Although the experimental study by Seavey ${ }^{10}$ for abrasive blasting in excess of $100 \mathrm{psi}$ at the nozzle, shows that by increasing the pressure from $60 \mathrm{psi}$ to $140 \mathrm{psi}$, productivity and efficiency continues to increase; however, as the discharge pressure increases, the fuel consumption increases as well to provide horsepower requirements for compressor system.

\section{Multiphase flow}

The two fluid model, Eulerian-Eulerian (E-E), can in principle be used to solve any multiphase flow regime, considering adequate closure relations for the momentum equation, are provided. However, EulerianLagrangian (E-L) model is suitable only for disperse flows. In the E-E approach, the flow variables are functions of space and time, and hence are represented as fields. In the $\mathrm{E}-\mathrm{L}$ method, particles are considered individually, and the position and velocity of each particle is only a function of time. Therefore, in E-L approach, Navier-Stokes equations are solved for the continuous phase (similar to E-E method). However, for the disperse phase positions and velocity of each particle is obtained from Newton's second law.

Granular materials that are created from crushing or mining operations are generally highly angular or plastic abrasive media usually in a cylindrical shape. However, most of computational analyses of multiphase granular flows are performed for perfect spheres. Some studies such as Vu-Quoc et al. ${ }^{11}$ and Džiugys and Peters ${ }^{12}$ have worked on elliptical shaped particles that are created from a combination of spheres, or the study by Hopkins and Shen ${ }^{13}$ which has worked on spherical and disk shaped particles.

Hou et al. ${ }^{14}$ have studied numerically inside and outside of the abrasive water jet nozzle. The Eulerian twophase model has been adopted to simulate flow fields. Although the results were acceptable, Hou et al. ${ }^{14}$ have also demonstrated that the Eulerian two-phase model is not able to correctly predict the velocity of solid phase.

Van der Hoef et al. ${ }^{15}$ have reviewed numerically gassolid fluidised bed on the basis of whether a Lagrangian or an Eulerian model is used for the gas or particulate flow. It is suggested that the Lagrangian-Lagrangian
(L-L) model, where both gas phase and solid phase are represented by particles, is useful only for gassolid flow at extremely small scales, in which the thermal fluctuations of the gas phase have an influence on the motion of the large particles (Brownian motion). On the other end of the scale is E-E approach, in which both gas phase and solid phase are solved based on continuum description. The interaction between the two phases is resolved by drag force correlations which depends on relative velocity of phases and volume fraction of the solid phase. The problem with this model is that it does not accurately model gasparticle and particle-particle interactions. To overcome this drawback, the E-L model (or discrete phase model (DPM)) has been proposed. Van der Hoef et al. ${ }^{15}$ have concluded that for gas-solid fluidised bed the DPM provides the best results and $\mathrm{E}-\mathrm{E}$ model can simulate fluidised beds only at engineering scales (height $1-2 \mathrm{~m}$ ), where particle size are at least $1 \mathrm{~mm}$.

Gas-solid multiphase flows are classified in two dense or dilute regimes. If the volume fraction of particles is less than $10^{-3}$, then the gas-solid is in dilute regime, and when the volume fraction of particles is greater than $10^{-3}$, the flow regime is dense. ${ }^{16}$ For dilute regimes with volume fraction less than $10^{-6}$ one way coupling (Fluid $\rightarrow$ Particle) is required and for dilute regimes with greater volume fraction two-way coupling is required (Fluid $\leftrightarrow$ Particle), while for dense regimes four-way coupling (Fluid $\leftrightarrow$ Particle $\leftrightarrow$ Particle) is essential.

\section{Supersonic nozzle}

Simulation of solid-propellant rockets with aluminium droplets has been performed by Najjar et al. ${ }^{17}$ in which burning of aluminium droplets generates aluminiumoxide smoke. Eulerian formulation for simulation of fine smoke particles has been used in conjunction with a Lagrangian formulation for the larger aluminium droplets.

The study by $\mathrm{Li}$ and $\mathrm{Li}^{18}$ has numerically analysed the behaviour of spray particles in the cold spray gun nozzle. Two-phase flow DPM relation along $k-\varepsilon$ turbulence model was used for simulations. CFD results show that the nozzle exit diameter has significant influence on the velocity of the particles. It is mentioned that the velocity of the particles will reduce significantly along the shock waves outside of the nozzle. It has been found out that the particle velocity will increase with increasing gas inlet temperature. This is shown by Dykhuizen and Smith ${ }^{19}$ as well, where in cold spray the velocity of the gas is derived by

$$
V_{g}=M \sqrt{\gamma R T_{g}}
$$


where $V_{g}$ and $T_{g}$ are velocity and temperature of the gas, repectively.

Two types of separation pattern can be observed in a supersonic nozzle, the free shock separation (FSS) and the restricted shock separation (RSS). In FSS flow separates from the nozzle wall due to oblique shock, and the separation zone continuously to exit a nozzle as a free jet. In RSS, the separation is restricted to a limited size and will reattach to a nozzle wall before exit of a nozzle. Both FSS and RSS can be observed in a nozzle at different operating conditions, which highly depends on shock boundary layer interactions.

\section{Swirl flow}

Swirling flow in choked de Laval nozzle was investigated numerically by Pandolfi. ${ }^{20}$ The swirling flow is achieved through surface located at the inlet of the nozzle. The time-dependent technique for twodimensional (2D) axisymmetric configurations has been deployed. The centrifugal forces due to the tangential velocity act in increasing the pressure at outer boundaries and in decreasing it at the inner boundaries.

Liu et al. ${ }^{21}$ have investigated supersonic flow inside adiabatic C-D which was introduced by two identical tangential inlets. The numerical simulations in this study were steady state, and all the flow parameters were time-independent. For boundary conditions, total pressure and temperature with critical mass flow rate were employed. For turbulence modelling Reynolds Stress Model (RSM) was used. It has been concluded that both the axial velocity and the tangential velocity increases and the swirling flow with a large centrifugal acceleration that is produced at the inlet can get through the shock wave at the throat up to the outlet of the nozzle.

A free vortex type swirling flow in a long-circular pipe was investigated experimentally by Kitoh. ${ }^{22}$ It has been shown that, based on the tangential velocity distribution, the flow has three regions: wall, annular and core. In the wall region, the only effect that appears is the centrifugal force destabilising. Therefore, the classical mixing length model can predict the flow in wall region. The annular region is characterised by a flow skewness. Tangential velocity in core region was expressed as a sum of forced and free vortex motions. It is difficult to use an analytical approach to predict flow features in this region. It was suggested that using the RSM which can handle anisotropic turbulence will be a more promising tool to predict the flow compared with an eddy viscosity model such as $k-\varepsilon$. The core region is characterised by a forced vortex motion, and the flow is dependent upon the upstream conditions. In this region, turbulence motion has very low frequency, and the flow is non-dissipative. Hence, there is a long history effect in core region.

\section{Numerical methods}

\section{Geometry}

The dimension of the nozzle, for which the flow inside it has been investigated, is shown in Figure 1(a); the length of the nozzle is $200 \mathrm{~mm}$ and has three sections. The first $(64 \mathrm{~mm})$ is the convergence section; then it is $16 \mathrm{~mm}$ with constant $11 \mathrm{~mm}$ diameter; and the divergence section length is $120 \mathrm{~mm}$ with outlet diameter of
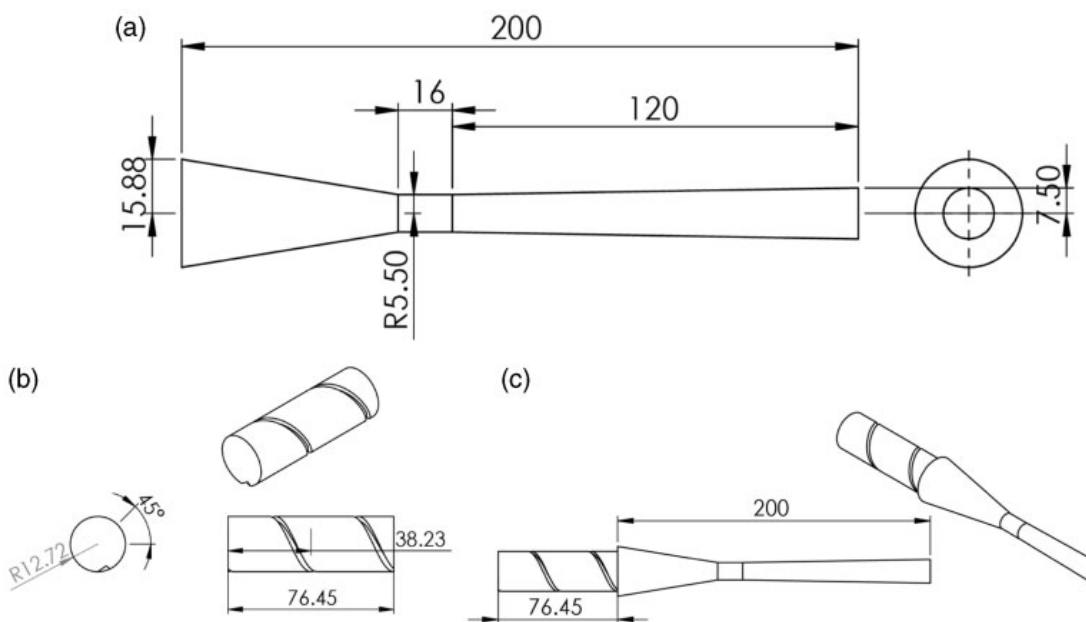

(c)

Figure I. Geometry of: (a) the nozzle; (b) the helical insert; and (c) nozzle and helical insert (all geometries shown here are with zero thickness). 
$15 \mathrm{~mm}$. This is one of standard geometries, which is being used widely by sandblast companies.

In order to create swirl flow inside the nozzle, a helical insert has been added to the inlet of the nozzle. The geometry is not expensive to manufacture, and it is easy to install on any sandblasting nozzle. The geometry of helical section is shown in Figure 1(b); it has $31.75 \mathrm{~mm}$ diameter with a length of $76.45 \mathrm{~mm}$, and the spiral part has two revolutions with start angle of $45^{\circ}$.

The computational domain is presented in Figure 1(c), where the helical insert and nozzle will screw together, with the total length of $276.45 \mathrm{~mm}$. All analyses are with same geometry but different pressure ratios.

\section{Governing equations}

The density-based scheme employs the density as a primary variable and extract pressure from the equation of state. The FLUENT package has been used in this study, the density-based solver, solves governing equations (continuity, momentum and energy) simultaneously as a set or vector of equations (equation (2)). For additional scalars, the governing equations will be solved sequentially (segregated) similar to pressure equation. The Navier-Stokes equations for compressible flow in vector mode can be written in conservation form as ${ }^{23}$

$$
\frac{\partial}{\partial t} \int_{V} \mathbf{W} \mathrm{d} V+\oint[\mathbf{F}-\mathbf{G}] \cdot \mathrm{d} A=\int_{V} \mathbf{H} \mathrm{d} V
$$

where vector $\mathbf{H}$ is the source term, and vectors $\mathbf{W}, \mathbf{F}$ and $\mathbf{G}$ are defined as

$\mathbf{W}=\left\{\begin{array}{c}\rho \\ \rho u \\ \rho v \\ \rho w \\ \rho E\end{array}\right\}, \quad \mathbf{F}=\left\{\begin{array}{c}\rho v \\ \rho v u+P \hat{i} \\ \rho v v+P \hat{j} \\ \rho v w+P \hat{k} \\ \rho v E+P v\end{array}\right\}, \quad \mathbf{G}=\left\{\begin{array}{c}0 \\ \tau_{x i} \\ \tau_{y i} \\ \tau_{z i} \\ \tau_{i j} v_{j}+q\end{array}\right\}$

Here, $e_{t}$ is total energy per unit mass, and $q$ is the heat flux. The inviscid flux vector $\mathbf{F}$ is evaluated by upwind, flux differencing splitting, ${ }^{24}$ where discrete flux at each face is obtained by

$$
F_{f}=\frac{1}{2}\left(F_{L}+F_{R}\right)-\frac{1}{2} \sum \alpha_{k}\left|\lambda_{k}\right| e_{k}
$$

where $e_{k}$ are the right eigenvectors of Jacobian matrix of $\partial \mathbf{F} / \partial \mathbf{W}, \alpha_{k}$ is the strength of $k$ th wave, and $\lambda_{k}$ is the eigenvalue of $\partial \mathbf{F} / \partial \mathbf{W}$, which represents the velocity of $k$ th wave. The fluxes are computed on left $\left(F_{L}\right)$ and right $\left(F_{R}\right)$ hand side of the face.
The partial differential equations are solved in transient mode with implicit density-based solver. The discretisation method for the gradient is least squares, and for flow is third-order MUSCL scheme to capture shock wave properties.

Discrete phase model. In DPM, each discrete phase element is tracked through flow domain. The trajectory of particles is obtained by solving Newton's second law of motion for each particle

$$
\begin{gathered}
m_{D} \frac{\mathrm{d} \mathbf{u}_{D}}{\mathrm{~d} t}=\sum \mathbf{F}_{D} \\
I_{D} \frac{\mathrm{d} \omega_{D}}{\mathrm{~d} t}=T_{D}
\end{gathered}
$$

where $m_{D}$ is the mass of dispersed phase, $\sum \mathbf{F}$ is all forces acting on particle, $I_{D}$ is the moment of inertia of particle, $\omega_{D}$ is the angular velocity of particle, and $T$ is the torque arising from tangential component of contact forces on particle. ${ }^{25}$ There are different type of forces acting on individual particles, depending on the nature of flow, but for complicated flow regimes are still not fully understood. In this research and for high Reynolds number flows, equation (5) can be shown as

$$
\begin{aligned}
m_{D} \frac{\mathrm{d} u_{D}}{\mathrm{~d} t}= & m_{D} \mathbf{F}_{d}\left(u_{C}-u_{D}\right)+m_{D} \frac{\rho_{C}}{\rho_{D}}\left(\frac{D u_{C}}{D t}\right) \\
& +\mathbf{g} m_{D}\left(1-\frac{\rho_{C}}{\rho_{D}}\right)
\end{aligned}
$$

where $\mathbf{g}$ is the gravitational acceleration, and $\mathrm{u}_{C}$ is the speed of the continuous phase. The right-hand side of equation (7) represents the drag force due to relative motion, the force due to pressure gradient and viscous stresses and the buoyancy force, respectively. In multiphase flow, there are three types of forces acting on phases. Volume forces such as gravity, inertia and buoyancy force, surface forces such as pressure or viscous force, and line forces like surface tension force. For a particle immersed in a continuous fluid, there are pressure forces and viscous forces that are acting on a surface of a particle. Therefore, the resulting force (drag force) implemented by a surrounding fluid on a particle is closed integral of the pressure and viscous stresses acting on a particle surface. ${ }^{26}$ The drag force $F_{d}$ on sphere particles is ${ }^{27}$

$$
\begin{gathered}
F_{d}=\frac{3}{8} C_{d} \frac{\rho_{C}\left|\mathbf{u}_{C}-\mathbf{u}_{D}\right|}{\rho_{D} r_{D}} \\
C_{d}=\frac{24}{R e}\left(1+\frac{R e^{2 / 3}}{6}\right) \\
R e \equiv \frac{2 \rho_{C}\left|\mathbf{u}_{C}-\mathbf{u}_{D}\right|}{\mu}
\end{gathered}
$$


where $\mu$ is the molecular viscosity, $r_{D}$ is the radius of particle, and $R e$ is the Reynolds number. Volume and line forces are negligible for this research.

The difficulty in Lagrangian approach lies in the fact that the continuous and dispersed phases are closely coupled. So, the continuous phase flow will affect the motion and distribution of particles, and also particles will influence the flow characteristics of continuous phase. The motion of continuous phase is described in an Eulerian framework (fixed in space), while the motion of particles is described in a Lagrangian framework (fixed to particle). Therefore, in this research, two-way coupling is applied to simulations, where particles interact with continuous phase.

\section{Turbulence modelling}

The two equation turbulence models are incapable of capturing anisotropy of the normal stresses and computing the effect on the turbulence of extra strain and body forces. The RSM models ${ }^{28, a}$ consider all these effects by solving transport equations for the Reynolds stresses, together with an equation for the dissipation rate. This will add extra seven transport equations for three-dimensional (3D) flows. ${ }^{\mathrm{b}}$ On the downside RSM will increase the amount of storage and CPU usage run time significantly.

The exact transport equation of Reynolds stress $\left(\rho \overline{u_{i}^{\prime} u_{j}^{\prime}}\right)$ can be written as follows:

$$
\frac{\partial}{\partial t}\left(\rho \overline{u_{i}^{\prime} u_{j}^{\prime}}\right)+\frac{\partial}{\partial x_{k}}\left(\rho \overline{u_{k}} \rho \overline{u_{i}^{\prime} u_{j}^{\prime}}\right)=P_{i j}+D_{i j}-\epsilon_{i j}+\Pi_{i j}+\Omega_{i j}
$$

where $P_{i j}$ is the rate of production of Reynolds stress, $D_{i j}$ is transport of Reynolds stress by diffusion, $\epsilon_{i j}$ is the rate of dissipation, $\Pi_{i j}$ is the transport of Reynolds stress due to pressure-strain interaction, and $\Omega_{i j}$ is the transport of Reynolds stress by rotation. The production term is

$$
P_{i j}=-\left(\rho \overline{u_{i}^{\prime} u_{k}^{\prime}} \frac{\partial \overline{u_{j}}}{\partial x_{k}}+\rho \overline{u_{i}^{\prime} u_{k}^{\prime}} \frac{\partial \overline{u_{i}}}{\partial x_{k}}\right)
$$

The rotation term is formulated as

$$
\Omega_{i j}=-2 \omega_{k}\left(\overline{u_{j}^{\prime} u_{m}^{\prime}} \epsilon_{i k m}+\overline{u_{i}^{\prime} u_{m}^{\prime}} \epsilon_{j k m}\right)
$$

where $\omega_{k}$ is the rotation vector. The diffusion term is modelled as

$$
D_{i j}=\frac{\partial}{\partial x_{m}}\left(\frac{v_{t}}{\sigma_{k}} \frac{\partial}{\partial x_{m}}\left(\rho \overline{u_{i}^{\prime} u_{j}^{\prime}}\right)\right)
$$

with $\sigma_{k}=1.0$. The kinematic viscosity is defined as

$$
v_{t}=C_{\mu} \frac{k^{2}}{\varepsilon} \quad C_{\mu}=0.09
$$

The dissipation rate of turbulence is calculated by

$$
\begin{aligned}
\varepsilon_{i j} & =\frac{(2 / 3) \rho \varepsilon \delta_{i j}}{v \frac{\partial u_{i}^{\prime}}{\partial x_{k}} \frac{\partial u_{j}^{\prime}}{\partial x_{k}}} \\
\varepsilon &
\end{aligned}
$$

The scalar dissipation rate is modelled with the same equation of standard $k-\varepsilon$ model

$$
\begin{aligned}
\frac{D \varepsilon}{D t}= & \frac{1}{\rho} \frac{\partial}{\partial x_{j}}\left[\frac{\mu_{t}}{\sigma_{\varepsilon}} \frac{\partial \varepsilon}{\partial x_{j}}\right]+\frac{C_{1} \mu_{t}}{\rho} \frac{\varepsilon}{k}\left(\frac{\partial \overline{u_{i}}}{\partial x_{j}}+\frac{\partial \overline{u_{j}}}{\partial x_{i}}\right) \frac{\partial \overline{u_{i}}}{\partial x_{j}} \\
& -C_{2} \frac{\varepsilon^{2}}{k}
\end{aligned}
$$

Second-order upwind discretisation scheme has been adopted for turbulence modelling.

The boundary layer is almost laminar in viscous sublayer and fully turbulent in log-law region. Turbulence models are valid for fully turbulent flows, therefore semi-empirical formulas 'wall functions', are used to model flow properties between the wall and log-law region. Reynolds numbers in this study are reasonably high, so using wall functions will provide proper accuracy without limiting computing resources.

\section{Results}

As explained previously, Eulerian model is not yet capable of accurately solving the interaction between fluid (air) and solid (particles) in such a high-speed flow and low-volume fractions $(<10 \%)$. On the other hand, Lagrangian method ${ }^{\mathrm{c}}$ is accurate and well tested for solid dynamic calculations. ${ }^{29}$

There are different abrasive materials for abrasive blasting machines. In the last couple of years sandblasting companies have put a lot of effort into providing environmentally friendly media to clean surfaces. These days there are two main media used by industry: crushed glass and olivine; particles in this research are considered as granular olivine particles. Abrasive particles that are used in the sandblasting industry have standard size. ${ }^{30}$ The major size used for most of the applications is particles with $0.01-0.15 \mathrm{~mm}$ diameter. Although the particles in reality are not spherical shape, but because of the size of particles that has been studied, and also the fact that the attention is not on studying the collision of particles on a surface, the geometry of particle will not have huge impact on simulation results. Hence, the simulations are based on 
Table I. Olivine properties.

\begin{tabular}{ll}
\hline Specific density & $3.32 \mathrm{~g} / \mathrm{cm}^{3}$ \\
Bulk density & $1.6-1.9 \mathrm{~g} / \mathrm{cm}^{3}$ \\
Melting point & $1600{ }^{\circ} \mathrm{C}$ \\
Thermal expansion & $0.0083 \mathrm{I} / \mathrm{K}$ \\
Thermal conductivity & $0.0025 \mathrm{cal} / \mathrm{s}-\mathrm{cm}$ \\
Hardness & 6.5 Moh's \\
\hline
\end{tabular}

spherical particles with $10 \mu \mathrm{m}(0.1 \mathrm{~mm})$ diameter with the density of $3200 \mathrm{~kg} / \mathrm{m}^{3}$. Olivine properties are explained in Table 1.

Sandblasting machines operate at different pressures and mass flow rates. The mass flow rate for abrasive particles could vary from 0.005 to $0.05 \mathrm{~kg} / \mathrm{s}$. In this study based on operation pressure (2-4 atm) and the nozzle dimension, the constant mass flow rate of olivine injection is considered to be $0.0211 \mathrm{~kg} / \mathrm{s}$. This is the average mass flow rate used in sandblasting companies. ${ }^{\mathrm{d}}$ The particles are injected uniformly from inlet surface (one particle from each computational node) with ambient temperature and zero velocity. All the DPM simulations are based on single phase simulation where unsteady tracking with fluid flow time steps has been performed on particles with spherical drag law and two-way coupling. Detailed analyses of single phase simulations on the same nozzle have been explained by Eslamian et al. ${ }^{6}$

\section{Inlet pressure $2 \mathrm{~atm}$}

Mach number contours at inlet pressure $2 \mathrm{~atm}$ for the nozzle without helical insert is shown in Figure 3. Compared with single phase simulation (Figure 2(a)), there is just one strong shock wave without any multiple shock and expansion wave after the main Mach disk. There is lower velocity area at the centre due to high concentration of sand (olivine) particles. The maximum velocity has reduced from Mach 1.8 to Mach 1.4, which means a $22 \%$ reduction. On the other hand, the maximum velocity of DPM with helical insert (Figure 4) has not changed compared with single phase simulation (Figure 2(b)) from Mach 1.6. The Mach number contours for swirl condition with particle injection shows that the structure of shock waves has not changed, and still there is series of shock waves and expansion fans after the main shock wave. However, the separation zone has changed, and there is a small FSS region at the top and an RSS region on the lower wall.

Particle distribution for the nozzle without helical insert is illustrated in Figure 5. Without swirl condition, the particles are more towards centre; however, by adding the swirl effect to the flow particles they are better distributed and are not concentrated at the centre of the nozzle (Figure 6). This will be very helpful to avoid damaging a surface.

Pressure along centre of the nozzle without helical insert is sketched in Figure 7. By injecting particles, the structure of shock waves has been changed. Instead of having multiple shock waves and expansion fans there is just one strong shock wave; after the pressure it will gradually increase until it reaches atmospheric pressure at the outlet. In Figure 8, the pressure along centre of the nozzle with helical insert is plotted. There is a very small change in pressure behaviour compared with single phase simulation, apart from some oscillations with very small amplitude before shock waves. There is a small movement on shock location as well, where first shock wave has moved forward.

\section{Inlet pressure $3 \mathrm{~atm}$}

Mach number contours with the enabled DPM for the nozzle without helical insert is represented in Figure 9. In comparison with single phase simulations, the maximum velocity has reduced by $13 \%$ from Mach 1.55 to Mach 1.77. However, in the nozzle with helical insert, the maximum velocity is almost unchanged at Mach 1.9 for both DPM (Figure 10) and single phase simulation (Figure 11). This proof there is much higher momentum in the swirling flow inside the nozzle even with the particles.

Figure 9 shows that there is some lower velocity zone at the centre of the nozzle due to high concentration of abrasive particles, but this is not visible in Figure 10 which verifies particles are not concentrated at the centre. Both non-swirl and swirl Mach contours with the Lagrangian approach do not show any unsteady pattern inside the domain.

From Figure 10 it is clear that both FSS separation zone at the top and RSS recirculation zone at the lower wall exist similar to single phase simulation (Figure 11), although these separation areas are much smaller. In the nozzle without helical insert, the separation zone has been removed due to particle injection.

Particle distribution for the nozzle without helical insert at inlet pressure $3 \mathrm{~atm}$ is very similar to inlet pressure $2 \mathrm{~atm}$, where most of olivine particles are concentrated at the centre of the nozzle in diverging section (Figure 12). Particles in the nozzle with helical insert are distributed more evenly, although there is some concentration zone after the helical insert, showed by red particles, due to the diameter difference between the nozzle inlet and helical insert outlet (Figure 13).

Pressure along centre of the nozzle without helical insert is plotted in Figure 14. In DPM, the shock wave is much weaker, and the pressure is gradually increasing to get to the atmospheric pressure at the outlet. This is not favourable effect as shock waves at the exit of the 


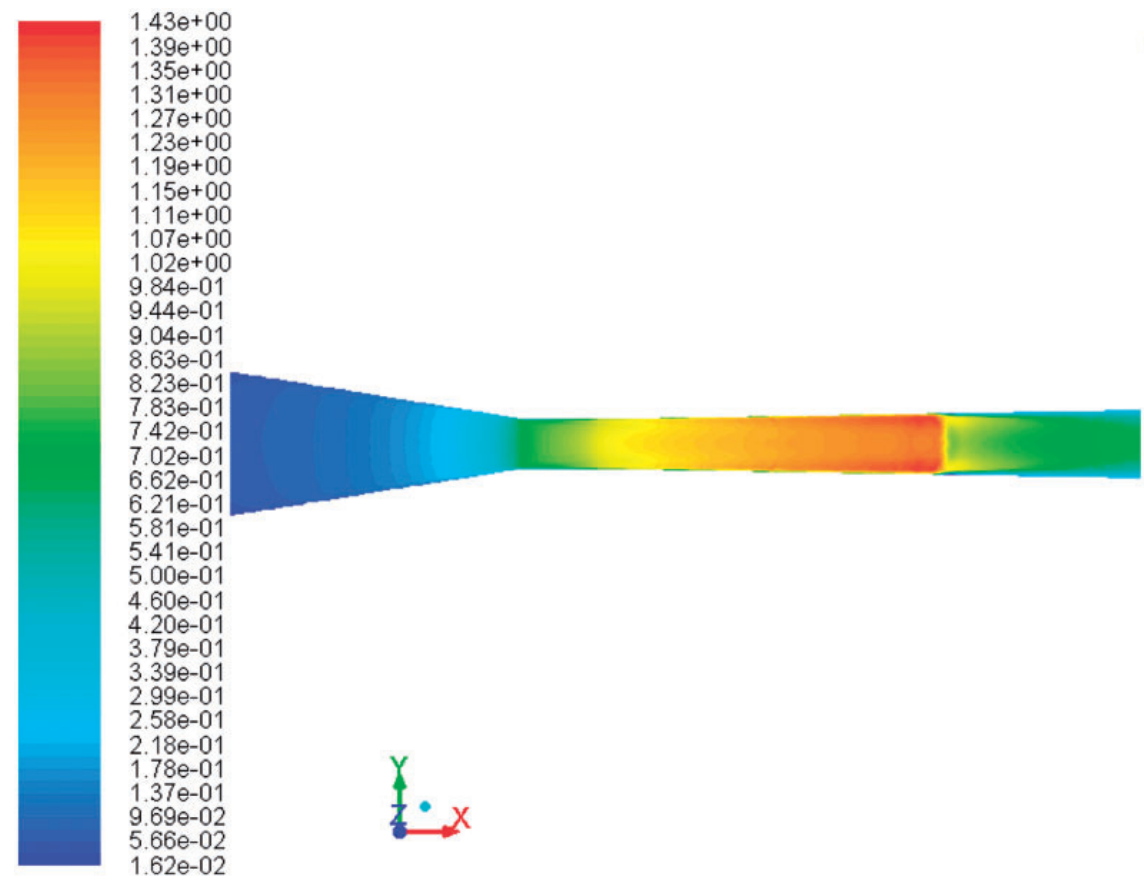

Figure 2. Mach number contours of DPM for the nozzle without helical insert at inlet pressure $2 \mathrm{~atm}$.

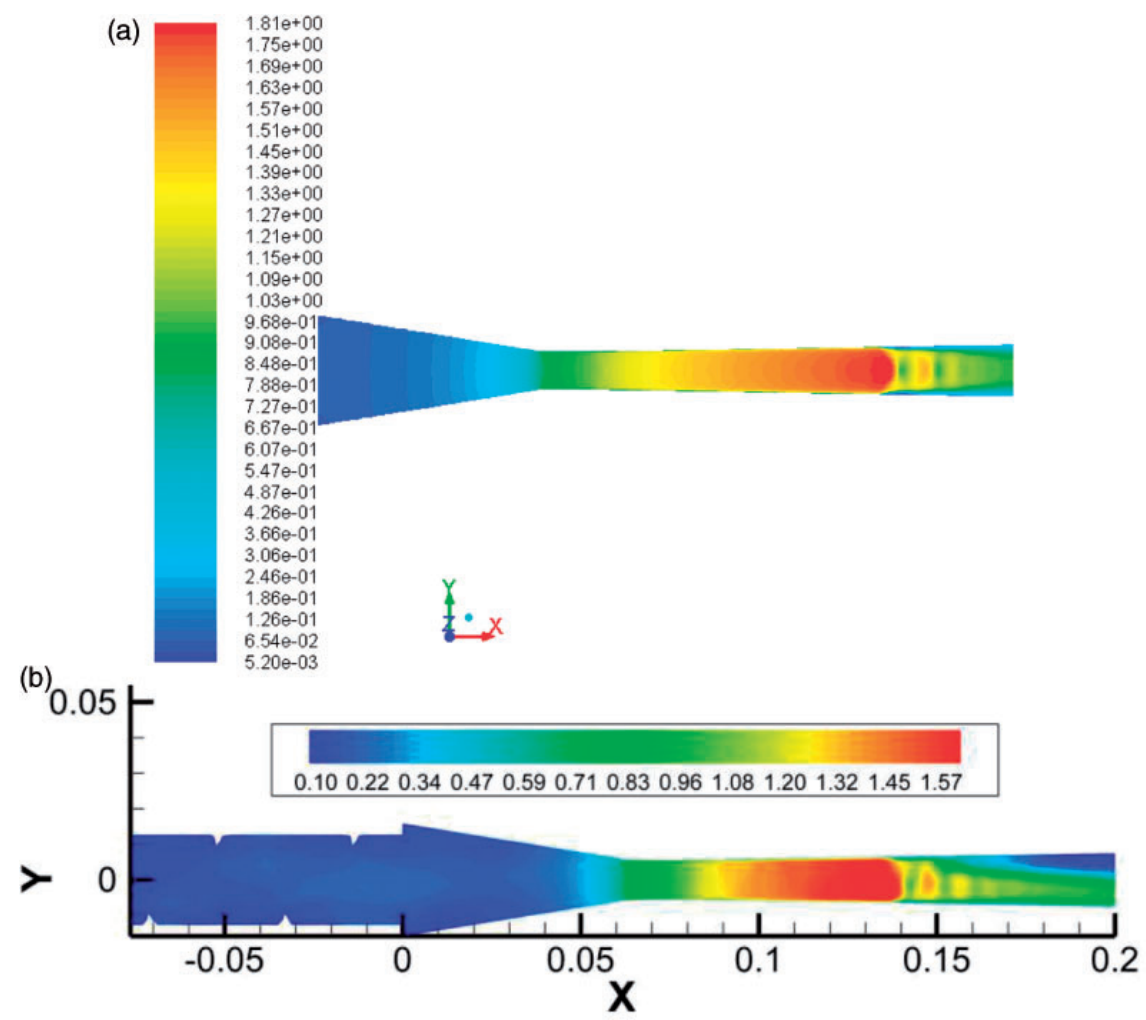

Figure 3. Mach number contours for single phase simulations of the nozzle: (a) without helical insert, and (b) with helical insert at inlet pressure 2 atm. $^{6}$ 


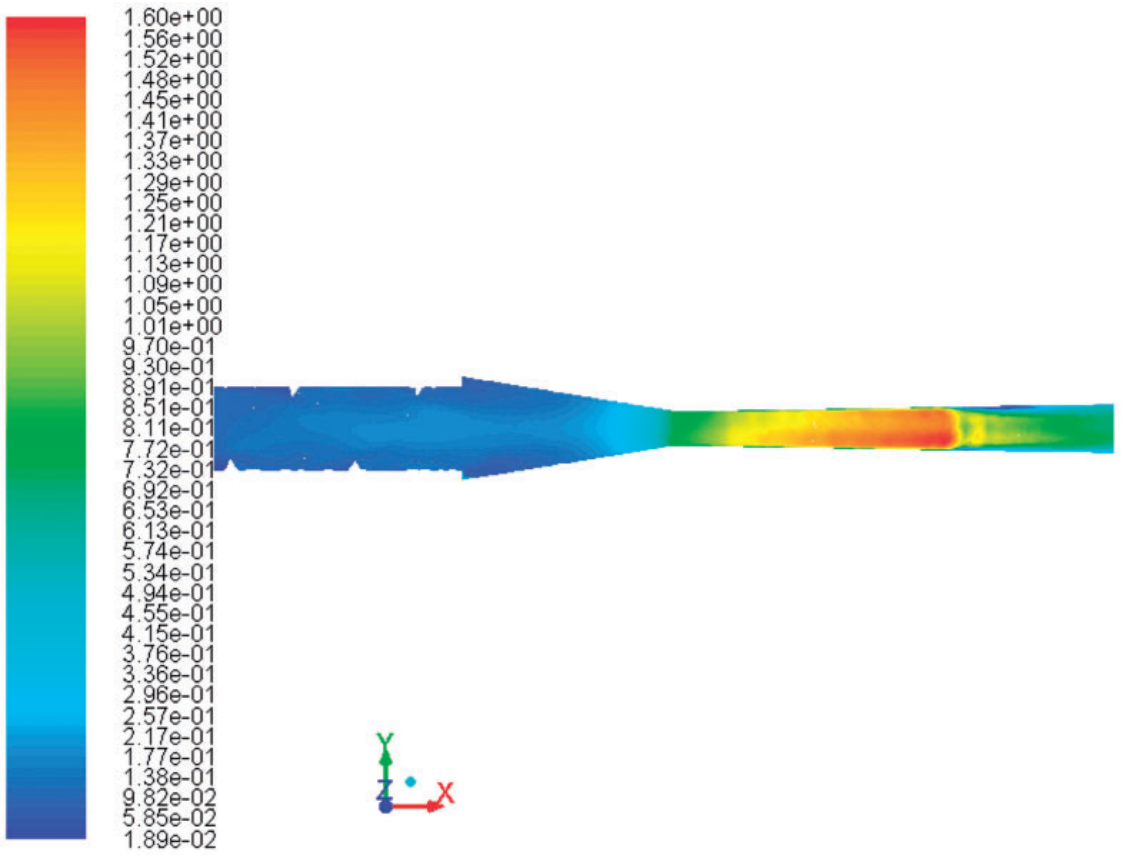

Figure 4. Mach number contours of DPM for the nozzle with helical insert at inlet pressure 2 atm.

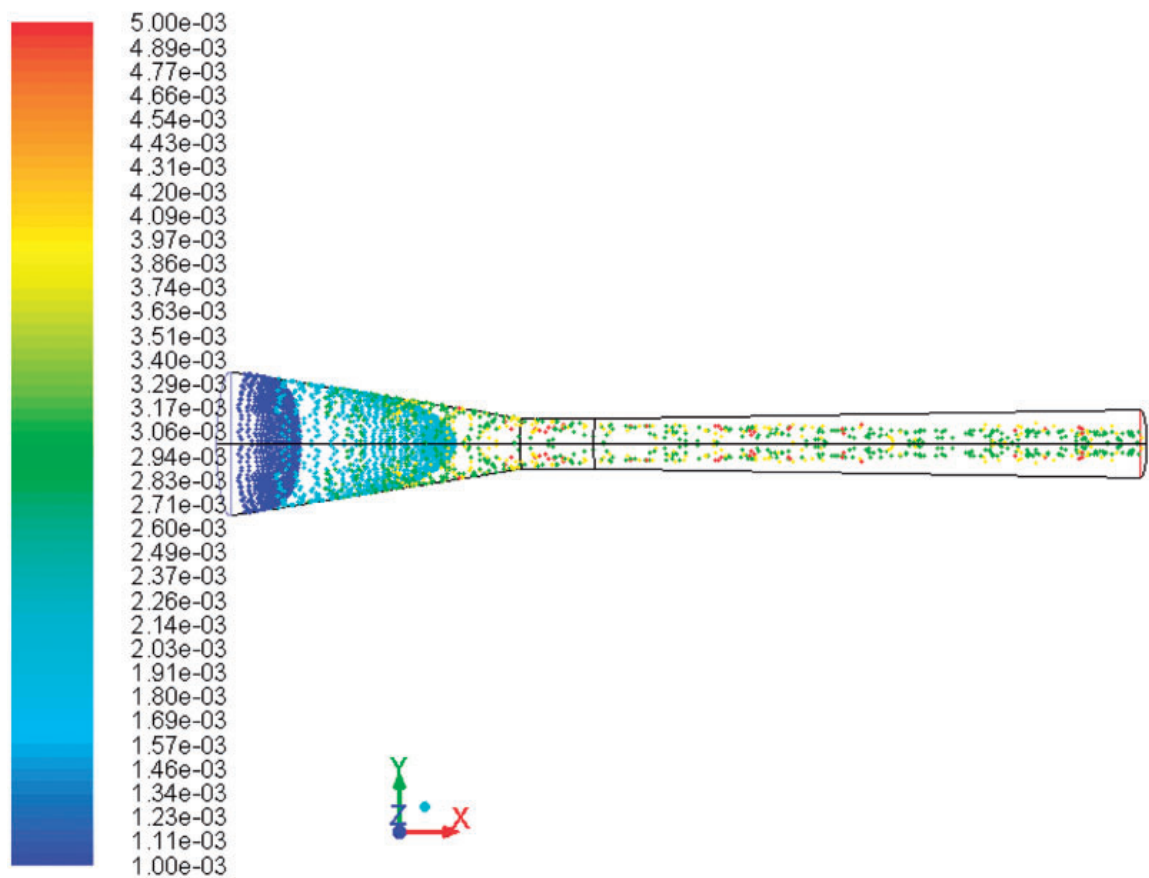

Figure 5. Particle trace coloured by particle residence time for the nozzle without helical insert at inlet pressure 2 atm.

nozzle will provide mixing effect. On the other hand for the nozzle with helical insert, the shock waves are almost unchanged (Figure 15). Similar to inlet pressure $2 \mathrm{~atm}$, the first shock wave has moved forward, and there are small oscillations behind the first shock.

\section{Inlet pressure $4 \mathrm{~atm}$}

Mach number contours for inlet pressure $4 \mathrm{~atm}$ are shown in Figures 16 and 17. In single phase simulation without helical insert, all the shock waves were outside of the nozzle (Figure 18), with the DPM as well, all the 


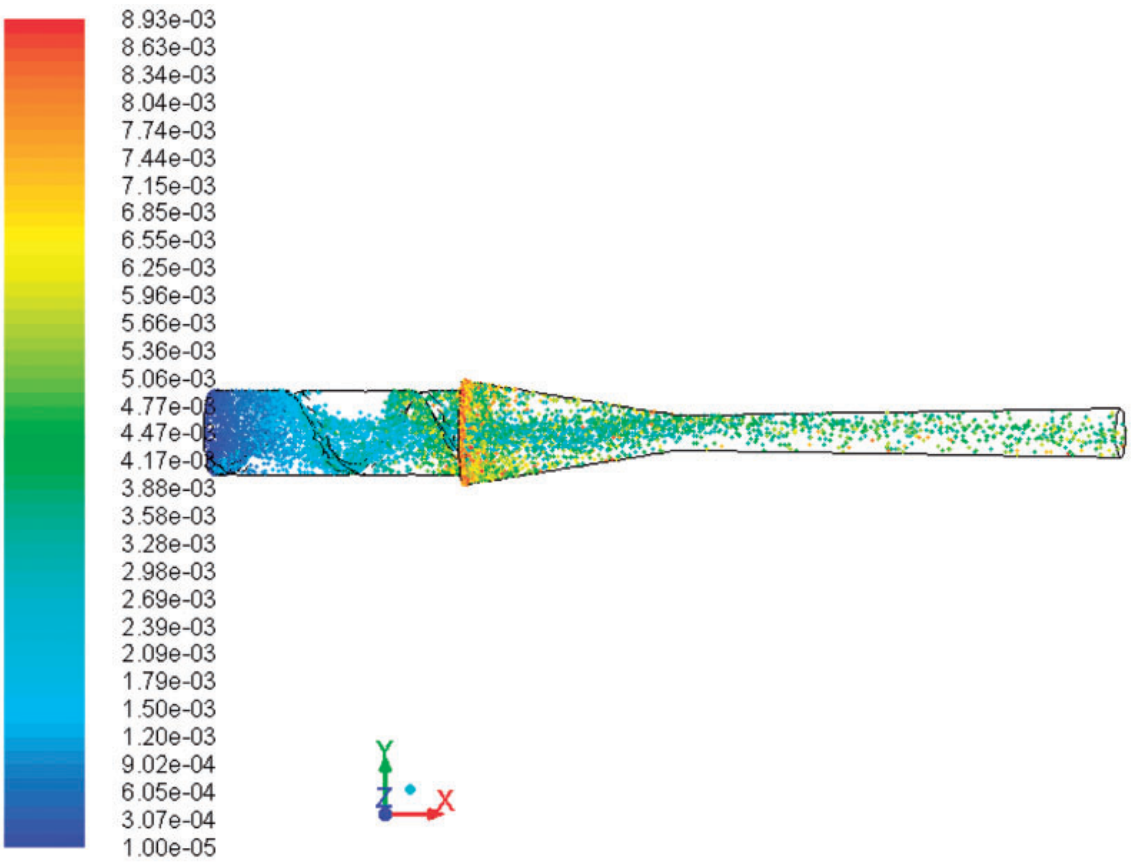

Figure 6. Particle trace coloured by particle residence time for the nozzle with helical insert at inlet pressure 2 atm.

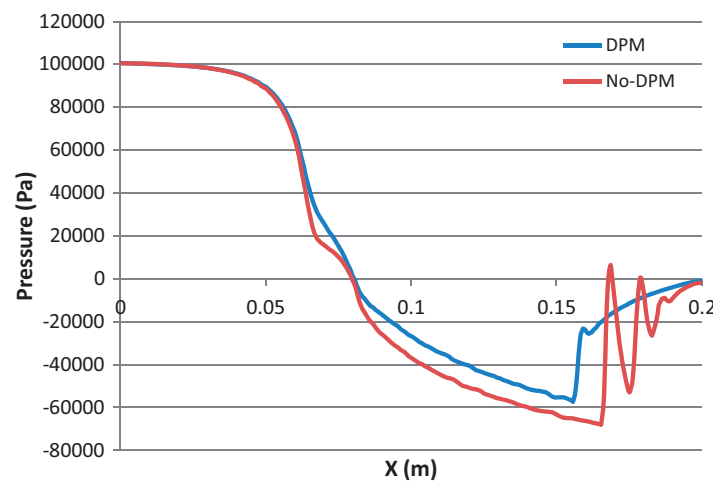

Figure 7. Pressure along centre of the nozzle without helical insert for DPM at inlet pressure $2 \mathrm{~atm}$.

DPM: discrete phase model.

shocks are outside of the nozzle, but there is an $8 \%$ reduction on maximum velocity. Similar to lower pressure DPMs for the nozzle without the helical insert, the air closer to the nozzle wall has a higher velocity than the air toward the centre of the nozzle; however, at inlet pressure $4 \mathrm{~atm}$; Figure 16) the velocity change from centre toward the nozzle wall is smaller.

Mach contours of the DPM for the nozzle with helical insert (Figure 17) show similar behaviour to single phase simulations (Figure 18). Same as lower pressures there is not any loss of maximum velocity by injecting particles, which illustrates a great advantage of using swirl flow for abrasive blasting. Although the solver is

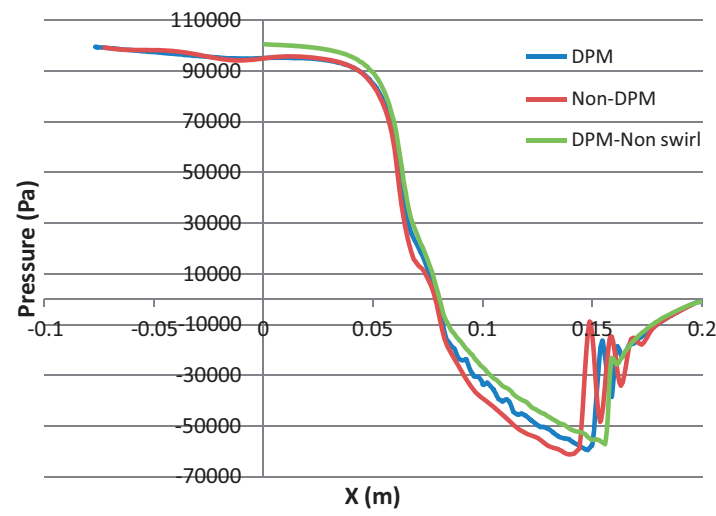

Figure 8. Pressure along centre of the nozzle with helical insert for DPM at inlet pressure $2 \mathrm{~atm}$.

DPM: discrete phase model.

transient and in single phase simulations showed unsteadiness in the flow, the DPM for the swirl flow does not demonstrate unsteadiness inside the nozzle. This could be because of a reduction on separation zone.

Particle distribution of inlet pressure $4 \mathrm{~atm}$ is different to that at lower pressures. For the nozzle without helical insert (Figure 19), unlike lower pressures explained above, particles are distributed more evenly although they are more toward centre of the nozzle. For the nozzle with helical insert (Figure 20), particles are more toward the upper wall up to the first shock 


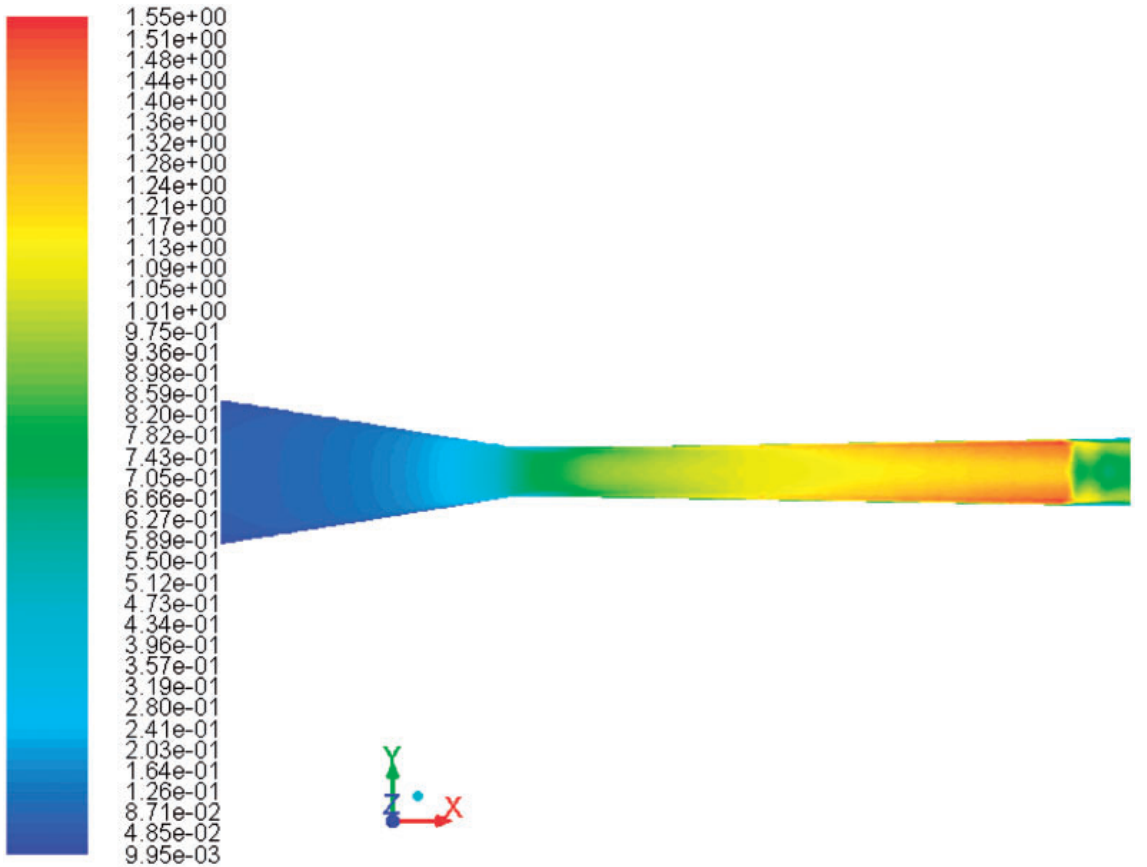

Figure 9. Mach number contours of DPM for the nozzle without helical insert at inlet pressure $3 \mathrm{~atm}$.
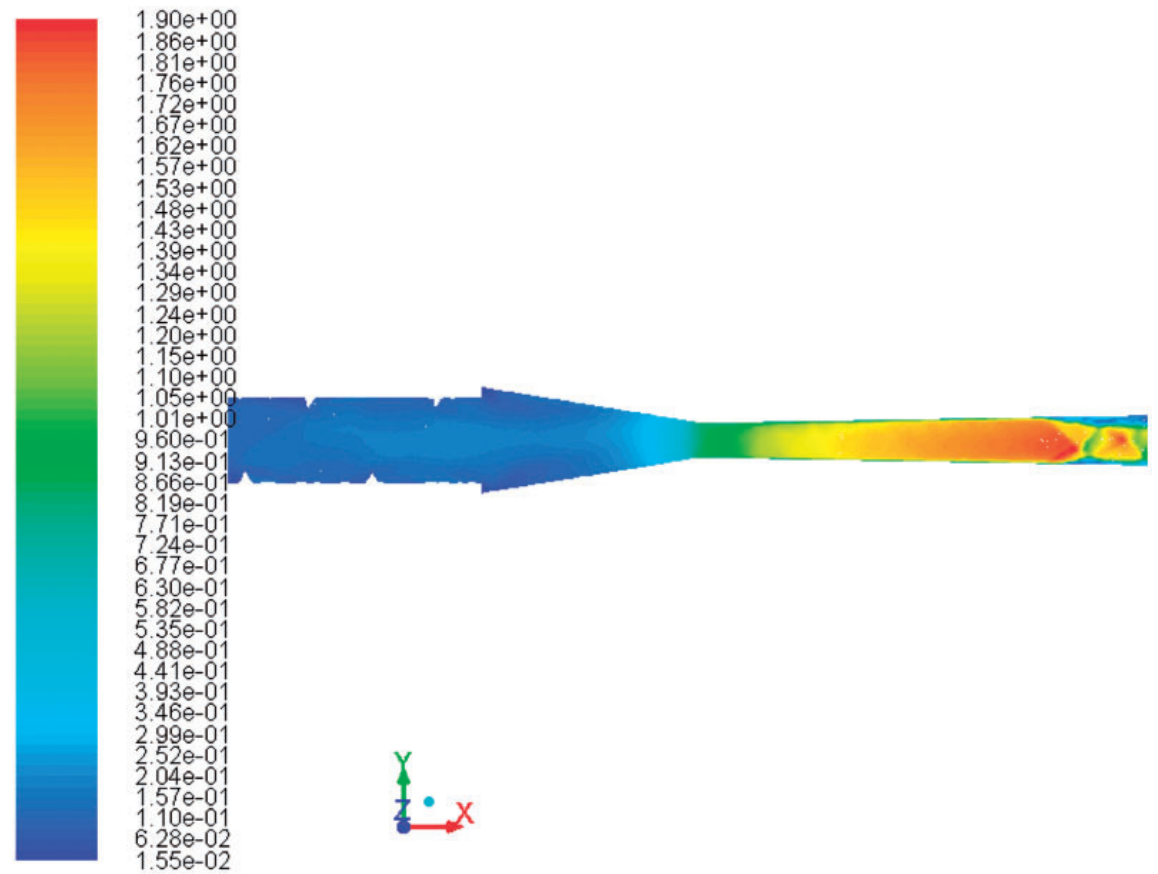

Figure 10. Mach number contours of DPM for the nozzle with helical insert at inlet pressure $3 \mathrm{~atm}$.

wave, and then they are distributed along the outlet as a mixing effect of shock wave.

Pressure along the centre line of the nozzle without helical insert (Figure 21) is almost identical to single phase simulation. This shows that the particles have less effect on pressure distribution inside nozzle but they have a major effect on shock wave structure as explained for lower inlet pressures. For the nozzle 


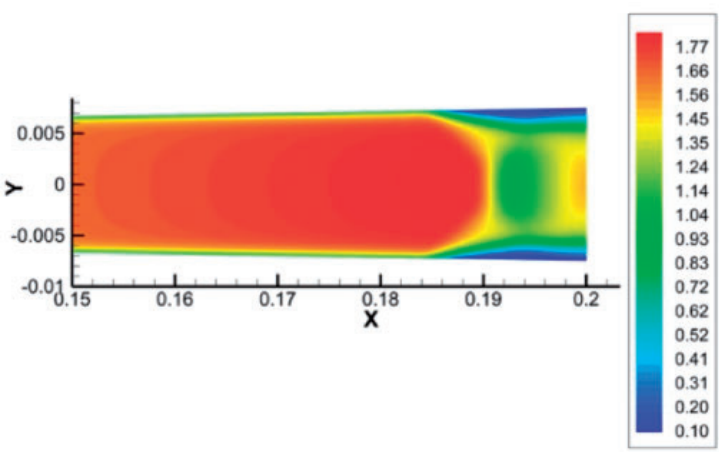

Non-swirl

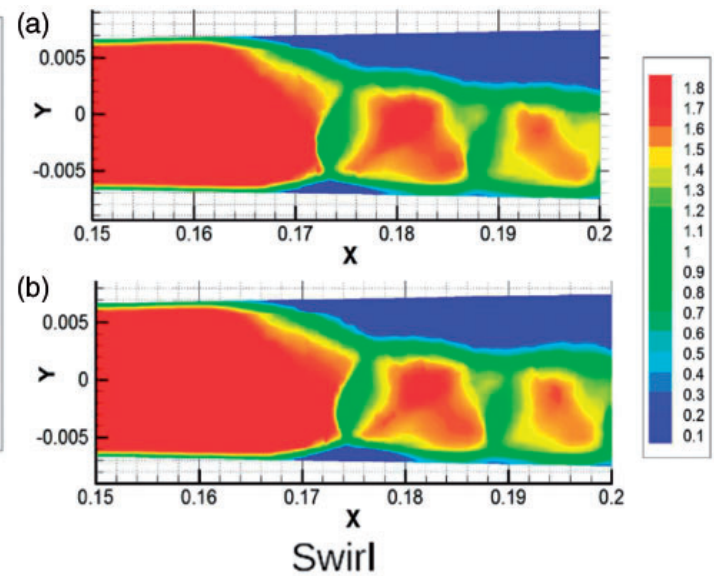

Figure II. Mach number contours for single phase simulations for inlet pressure $3 \mathrm{~atm}$. Swirl contours are for two time-steps: (a) $t=0.8 \mathrm{e}^{-2 \mathrm{~s}}$, and (b) $t=1.8 \mathrm{e}^{-2 \mathrm{~s}}$.
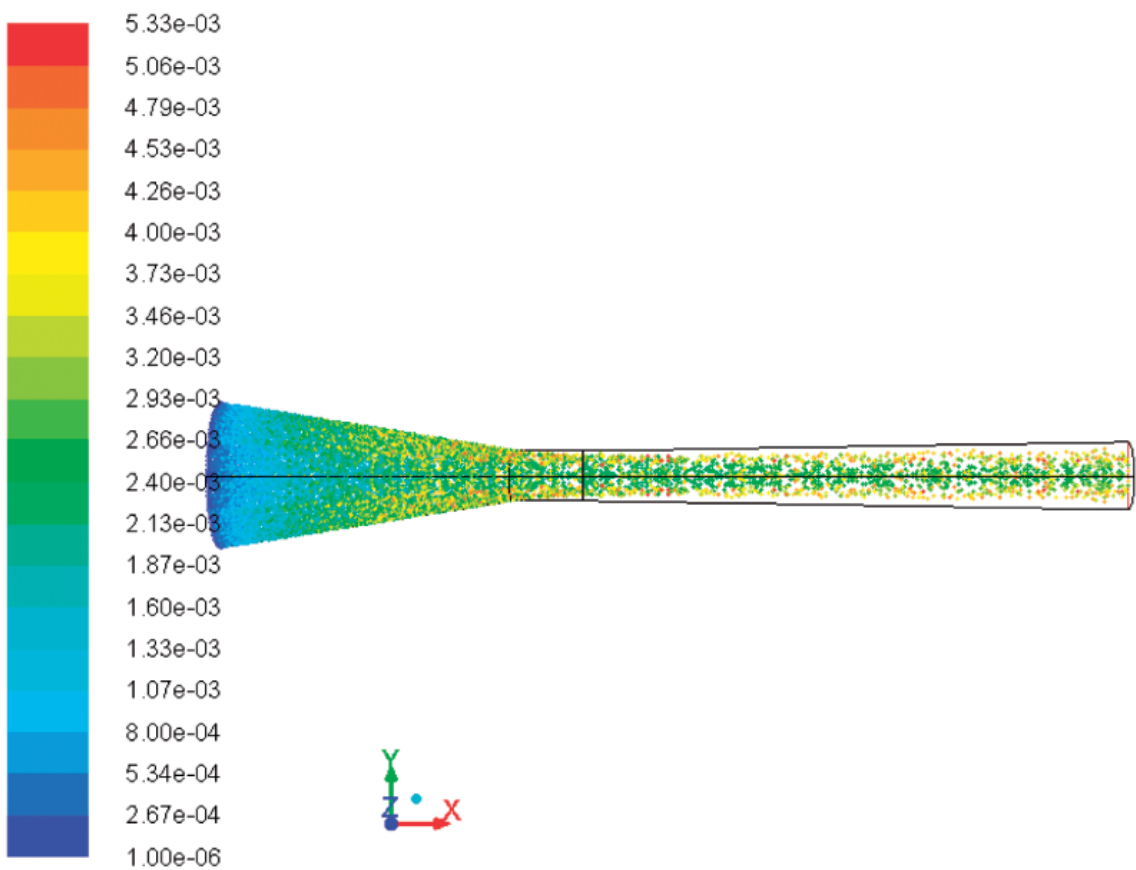

Figure 12. Particle trace coloured by particle residence time for the nozzle without helical insert at inlet pressure $3 \mathrm{~atm}$.

with helical insert (Figure 22), there is not any major change even in shock structure. Similar to lower pressures, there are small oscillations before the first shock wave in diverging section. Therefore, with the swirl effect, flow parameters will not see a major change between single phase simulations and Lagrangian simulations with particle injection.

\section{Verification and validation}

Grid dependency tests have been conducted on all cases, where extra grid refinement has not changed the results. As mentioned by Ferziger and Perić, ${ }^{31}$ it is important for the refinement to be substantial and systematic, as the systematic grid refinement studies are the most reliable and common studies. ${ }^{32}$ Systematic refinement means that the grid is refined in all directions with the same ratio. The grid dependency check has been performed by running simulations for $200 \mathrm{k}$, $320 \mathrm{k}$ and $500 \mathrm{k}$ cells. As the numerical results for cells above $320 \mathrm{k}$ were stable and similar, therefore, all the simulations are based on $320 \mathrm{k}$ cells with special attention in refining the mesh at the exit boundary to capture shock waves. 


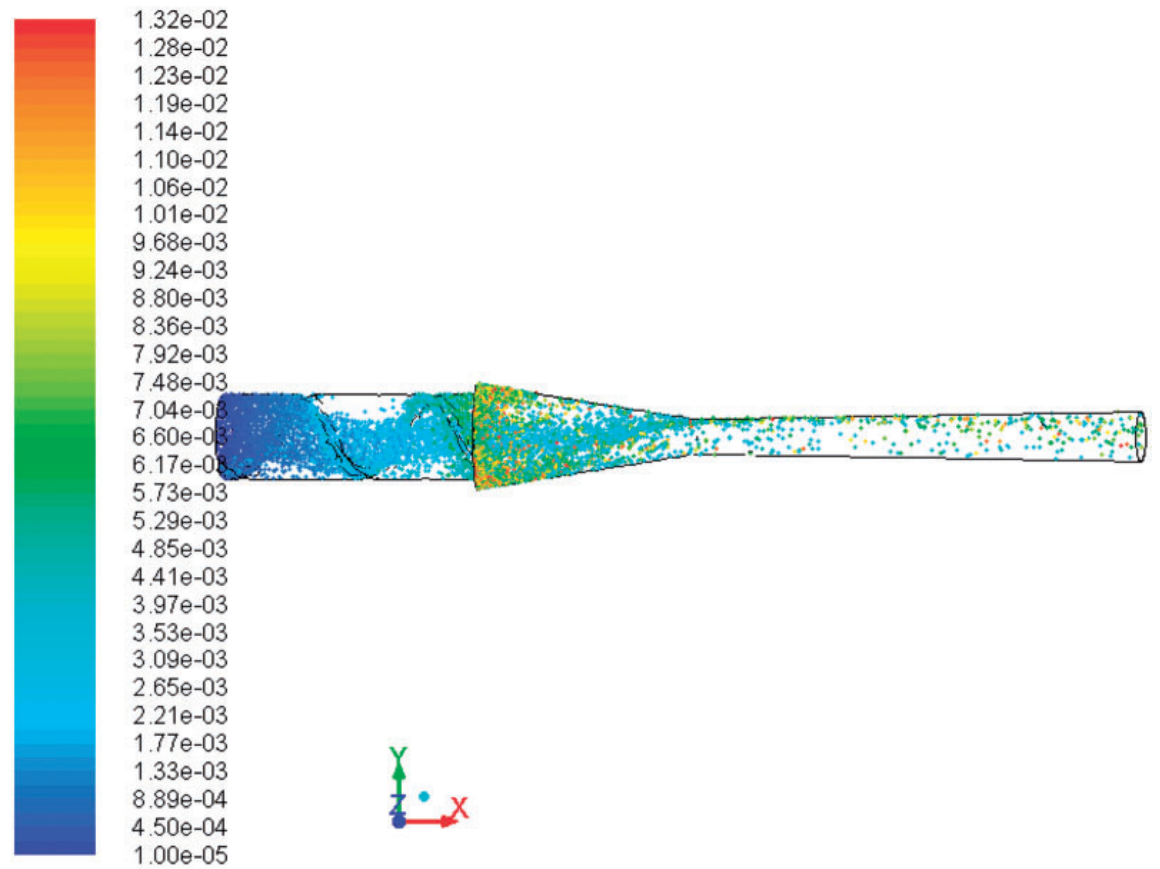

Figure 13. Particle trace coloured by particle residence time for the nozzle with helical insert at inlet pressure 3 atm.

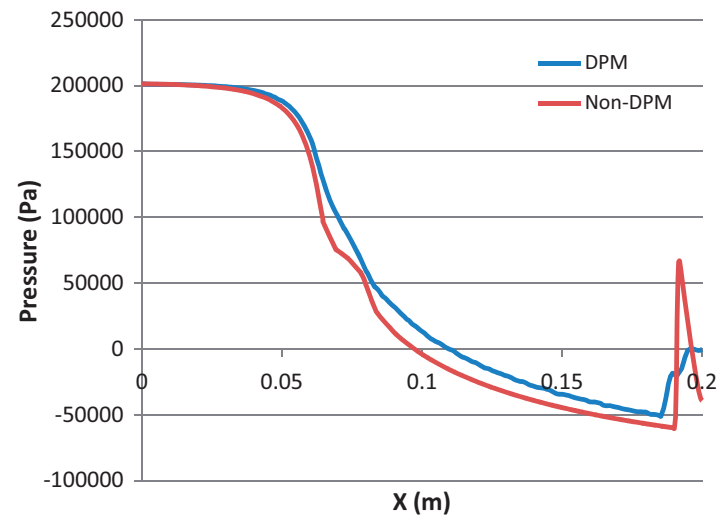

Figure 14. Pressure along centre of the nozzle without helical insert for DPM at inlet pressure $3 \mathrm{~atm}$. DPM: discrete phase model.

The maximum wall $y^{+}$values for the CFD simulations were 50, and the RSM which is a core-turbulent model gives better results for $y^{+}$values in the log-low region $\left(y^{+}>30\right.$ to 60$),{ }^{33}$ hence $\mathrm{y}^{+}$values were satisfied for most of the nozzle wall.

Validation is a process to assess numerical modelling uncertainty by using benchmark experimental data, and when it is possible, estimating the sign and magnitude of numerical modelling error. ${ }^{34}$ The fundamental strategy for validation is identification of the error and

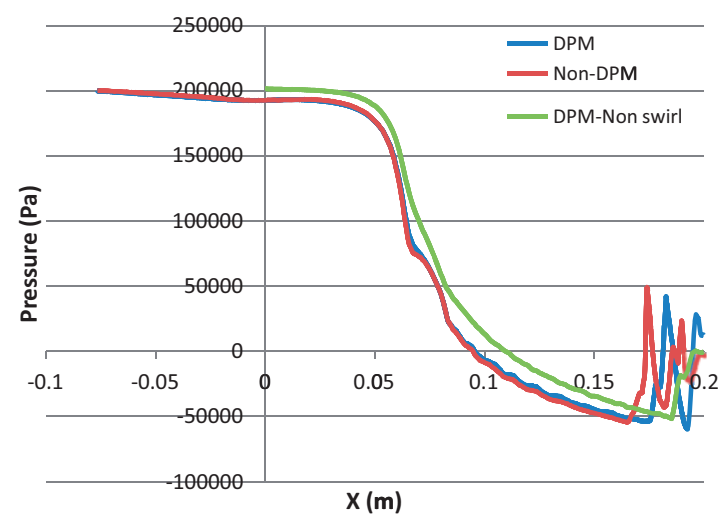

Figure I5. Pressure along centre of the nozzle with helical insert for DPM at inlet pressure $3 \mathrm{~atm}$.

DPM: discrete phase model.

uncertainty in the conceptual model, quantification of the numerical error in the numerical solutions, prediction for experimental uncertainty and finally a comparison between simulation results and experimental data. ${ }^{35}$ However, due to the impracticality and the difficulty of performing exact validation experiments on complex systems, recommended method is to use smallscale benchmark experiments. ${ }^{36}$

The numerical model was validated by running our model for same simulations of Xiao et al. ${ }^{37}$ The Mach 


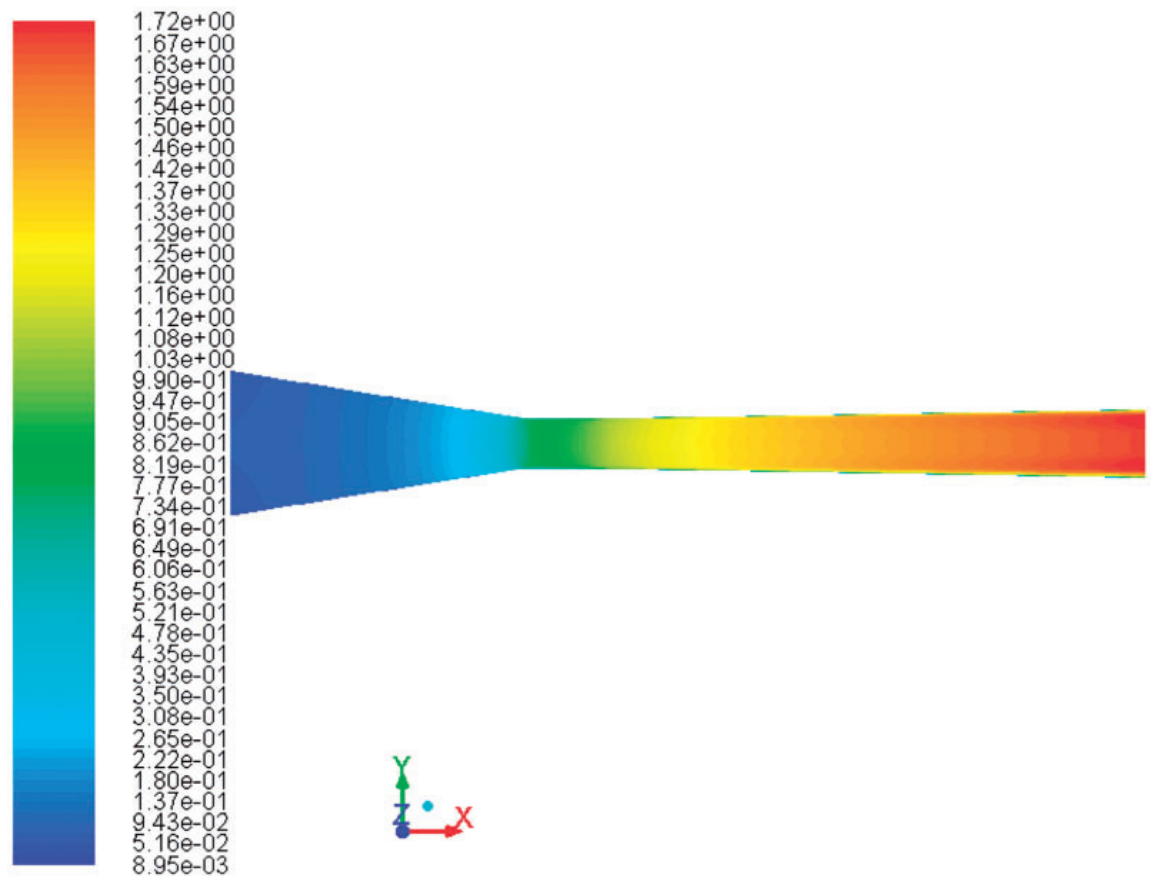

Figure 16. Mach number contours of DPM for the nozzle without helical insert at inlet pressure $4 \mathrm{~atm}$.
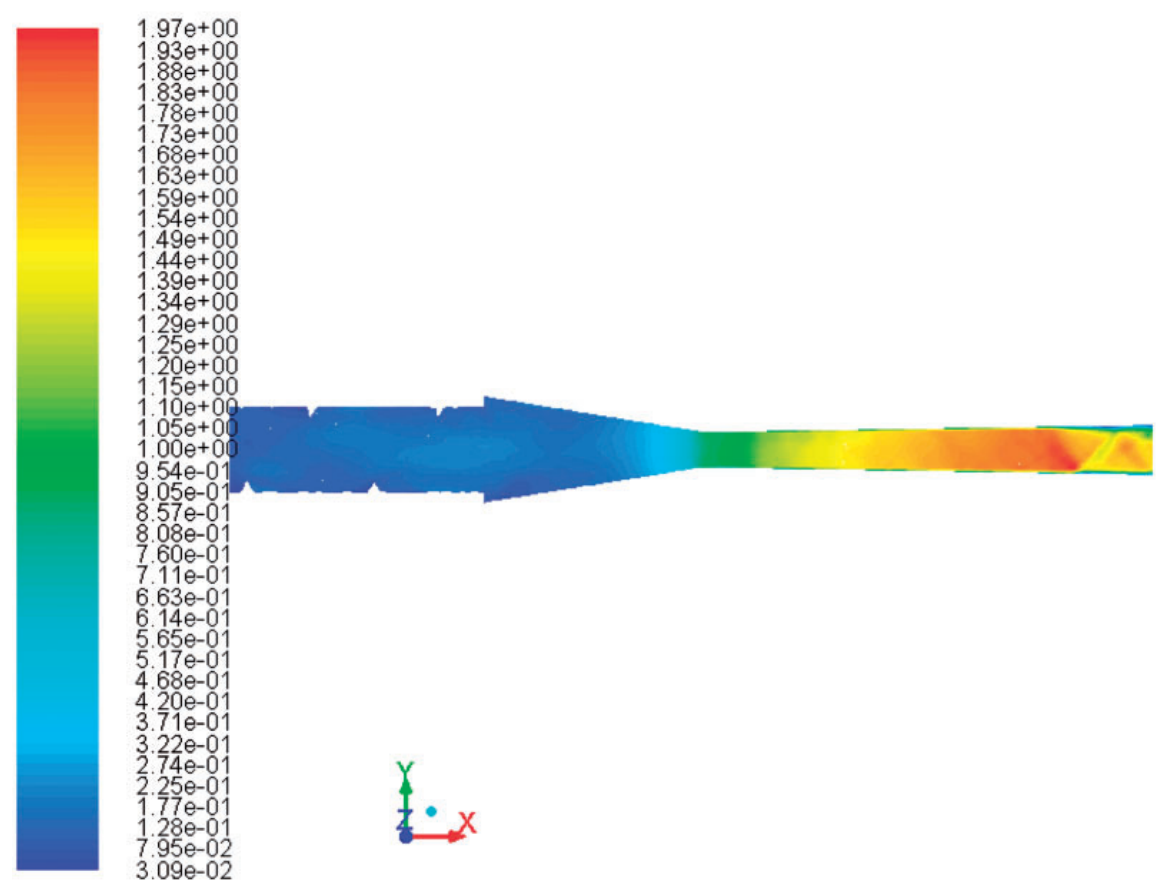

Figure 17. Mach number contours of DPM for the nozzle with helical insert at inlet pressure 4 atm. 


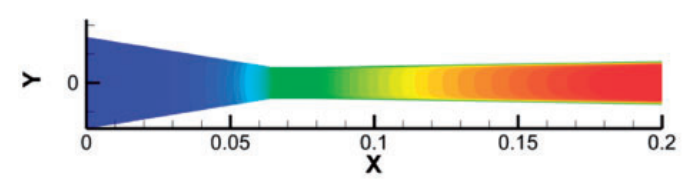

Non-swirl (a)

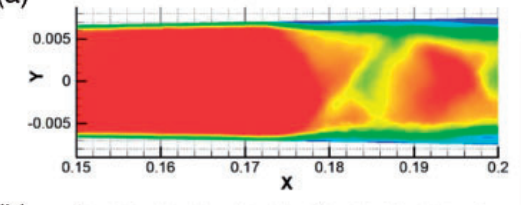

(b)

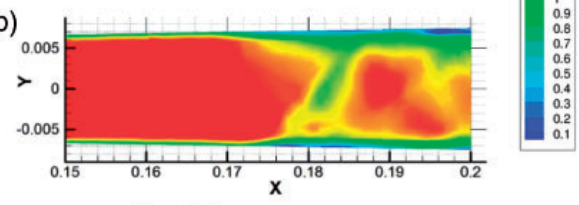

Swirl

Figure 18. Mach number contours for single phase simulations at inlet pressure $4 \mathrm{~atm}$. Swirl contours are for two time-steps: (a) $0.8 \mathrm{e}^{-2}$, and (b) $2.8 \mathrm{e}^{-26}$
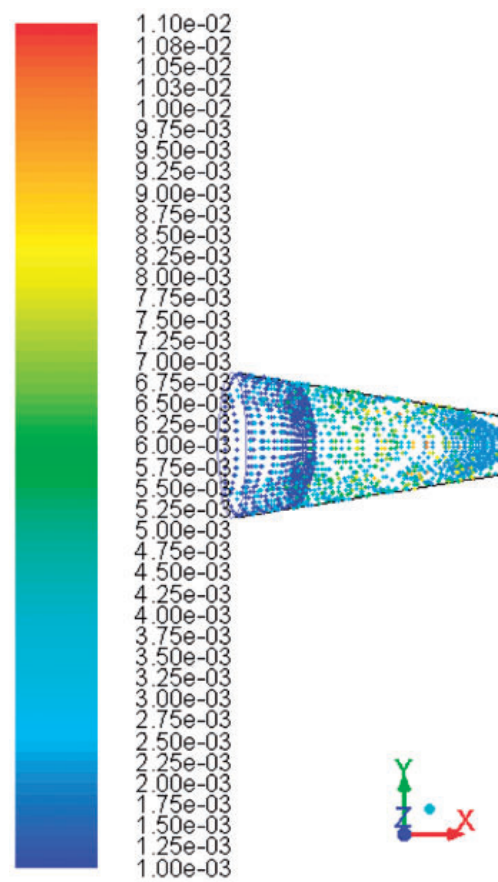

Figure 19. Particle trace coloured by particle residence time for the nozzle without helical insert at inlet pressure $4 \mathrm{~atm}$.

number contours for Fluent simulation at area ratio $\left(A_{e} / A_{t}\right) \quad 1.5$ and pressure ratio 2 are presented in Figure 23(b). The Mach contours are compared with Xiao et al. ${ }^{37}$ computational results (Figure 23(a)). These two numerical models are identical in all aspects. Both of $k-\omega$ and RSM turbulence models generated same results.

The centre line pressure is compared with experimental test by Abbasalizadeh et al. $^{38}$ and Abbasalizadeh. ${ }^{39}$ This experimental test was performed on the same nozzle without helical insert. Comparing experimental test with simulations (Figure 24), where both performed at inlet pressure $3 \mathrm{~atm}$, illustrates that there is good agreement between experimental data and numerical results. There is less than $3 \%$ error in calculations. This difference could be due to unsteady flow behind shock wave that creates a separation zone.

\section{Conclusion}

It was shown that Eulerian model is not sophisticated for granular supersonic flow unless there is a large volume fraction of the second phase. DPM results showed that maximum Mach number will be reduced by particles injection in the non-swirl nozzle, however, with swirl effect, there was not a major loss of 


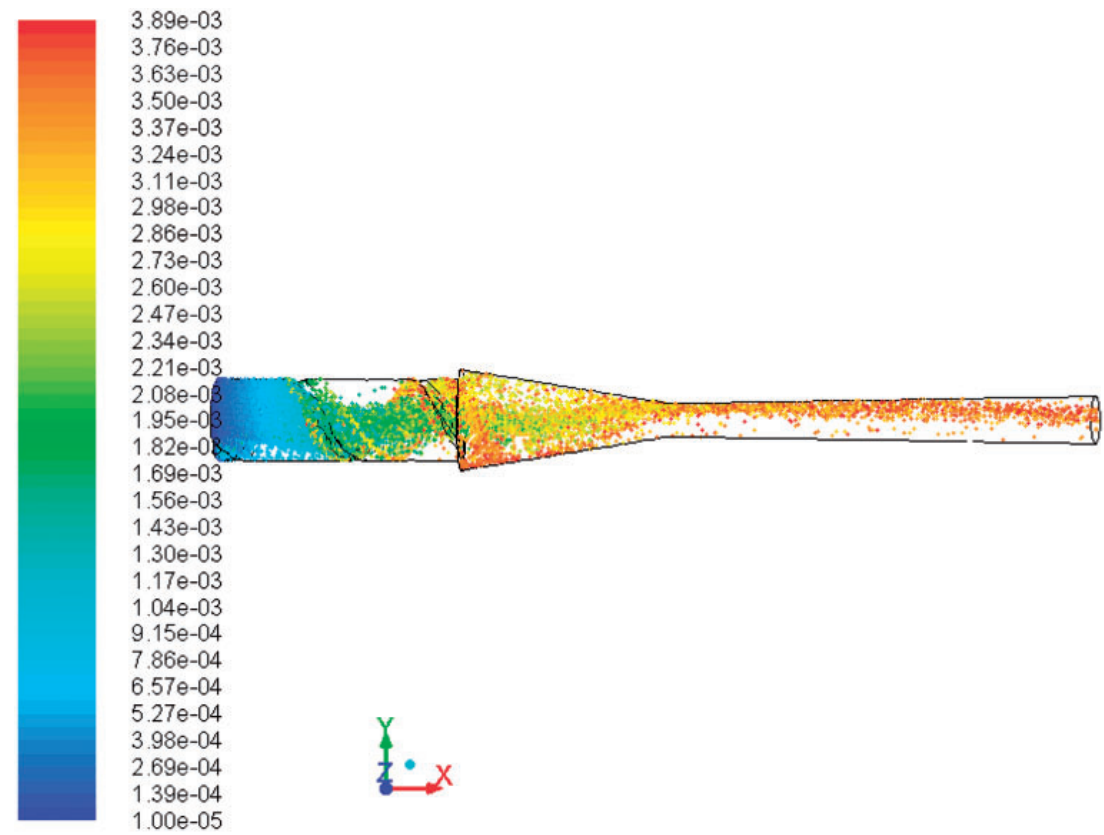

Figure 20. Particle trace coloured by particle residence time for the nozzle with helical insert at inlet pressure $4 \mathrm{~atm}$.

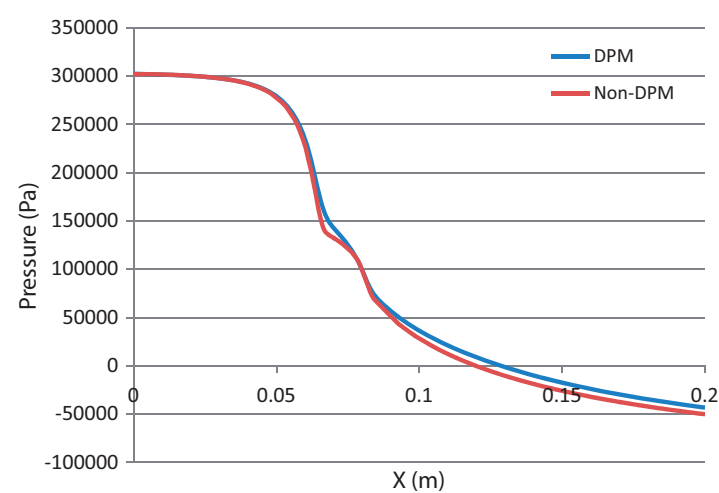

Figure 2I. Pressure along centre of the nozzle without helical insert for DPM at inlet pressure $4 \mathrm{~atm}$.

DPM: discrete phase model.

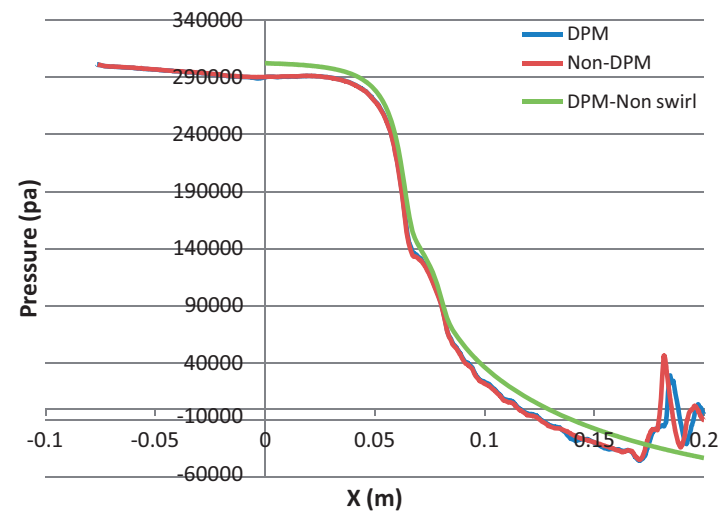

Figure 22. Pressure along centre of the nozzle with helical insert for DPM at inlet pressure $4 \mathrm{~atm}$.

DPM: discrete phase model.

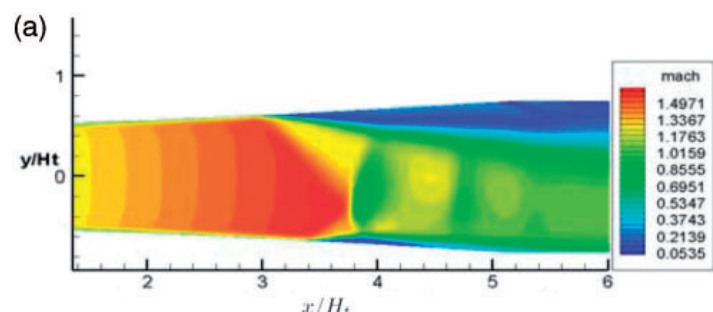

(b)

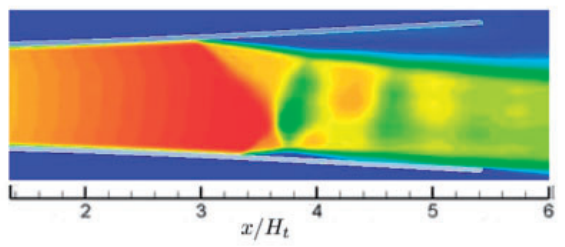

Figure 23. Mach number contours at $A_{e} / A_{t}=1.5$ and $P R=2.0$ for: (a) results by Xiao et al. ${ }^{37}$, and (b) CFD simulation.

maximum Mach number. This effect was more influential at lower pressure ratios where the maximum Mach number reduction was $22 \%$ at inlet pressure 2 atm and just $8 \%$ at inlet pressure $4 \mathrm{~atm}$. Unlike single phase simulations, in Lagrangian approach neither non-swirl nor swirl Mach number contours showed any unsteadiness inside the nozzle. This could be the result of reduction in size of separation zones. In DPM simulations for the nozzle without helical insert, as an effect of particles concentration at the centre of the nozzle, there were major changes on the shock wave structure. On the other hand, in DPM simulations for the nozzle with helical insert there were not any major change on the shock structure as there were better particle distribution 


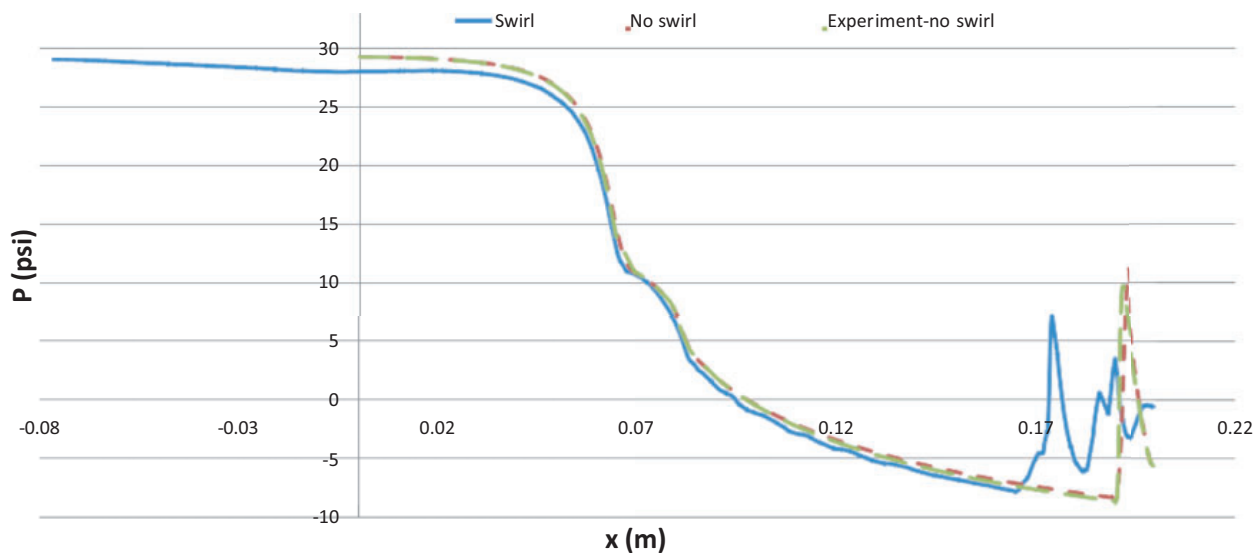

Figure 24. Static pressure at the centre of nozzle for inlet pressure $3 \mathrm{~atm}$.

inside the nozzle; also swirl flow increases turbulent intensity inside the nozzle.

\section{Declaration of conflicting interests}

The author(s) declared no potential conflicts of interest with respect to the research, authorship and/or publication of this article.

\section{Funding}

The author(s) received no financial support for the research, authorship and/or publication of this article.

\section{Notes}

a. Also called second-order closure model.

b. For two-dimensional flows this will be five extra equations.

c. Called DPM as well.

d. Our calculations are mainly based on data provided by Farrow System ${ }^{\circledR}$.

\section{References}

1. Anderson JD. Modern compressible flow with historical perspective. New York: McGraw-Hill, 2003.

2. Anderson JD. Hypersonic and high temperature gas dynamics. Reston, VA: American Institute of Aeronautics and Astronautics, 2000.

3. Syred N and Beér JM. Combustion in swirling flows: a review. Combust Flame 1974; 23: 143-201. ISSN: 00102180. http://dx.doi.org/10.1016/0010-2180(74)90057-1.

4. Schwartz IR. Minimization of jet and core noise of a turbojet engine by swirling the exhaust flow. In: Aeroacoustics conference, 2nd ed., Hampton, VA, 24-26 March 1975. Reston, VA: AIAA.

5. Lu HY, Ramsay JW and Miller DL. Noise of swirling exhaust jets. AIAA $J$ 1977; 15: 642-646.

6. Eslamian E, Shirvani H and Shirvani A. Numerical investigation of swirl flow inside a supersonic nozzle. Adv Fluid Mech IX 2012; 74: 131.
7. Deng J, Zhang X, Niu P, et al. Wear of ceramic nozzles by dry sand blasting. Tribol Int 2006; 39: 274-280. ISSN: 0301679X. http://dx.doi.org/10.1016/j.triboint.2004.07.026.

8. Jianxin D, Yihua F, Zeliang D, et al. Wear behavior of ceramic nozzles in sand blasting treatments. J Eur Ceram Soc 2003; 23: 323-329. ISSN: 0955-2219. http:// dx.doi.org/10.1016/S0955-2219(02)00183-8.

9. Bouzid $\mathrm{S}$ and Bouaouadja N. Effect of impact angle on glass surfaces eroded by sand blasting. J Eur Ceram Soc 2000; 20: 481-488. ISSN: 0955-2219. http://dx.doi.org/ 10.1016/S0955-2219(99)00140-5.

10. Seavey M. Abrasive blasting above 100 psi. J Protective Coat Linings 1985; 2: 26-37.

11. Vu-Quoc L, Zhang X and Walton O. A 3-d discreteelement method for dry granular flows of ellipsoidal particles. Comput Meth Appl Mech Eng 2000; 187: 483-528.

12. Džiugys A and Peters B. An approach to simulate the motion of spherical and non-spherical fuel particles in combustion chambers. Granul Matter 2001; 3: 231-266.

13. Hopkins MA and Shen HH. A Monte Carlo solution for rapidly shearing granular flows based on the kinetic theory of dense gases. J Fluid Mech 1992; 244: 477-491.

14. Hou RG, Huang CZ, Wang J, et al. Simulation of solidliquid two-phase flow inside and outside the abrasive water jet nozzle. Key Eng Mater 2007; 339: 453-457.

15. Van der Hoef MA, van Sint Annaland M, Deen NG, et al. Numerical simulation of dense gas-solid fluidized beds: a multiscale modeling strategy. Annu Rev Fluid Mech 2008; 40: 47-70.

16. Elghobashi S. On predicting particle-laden turbulent flows. Appl Sci Res 1994; 52: 309-329.

17. Najjar FM, Ferry JP, Haselbacher A, et al. Simulations of solid-propellant rockets: effects of aluminum droplet size distribution. J Spacecraft Rockets 2006; 43: 1258-1270.

18. Li W-Y and Li C-J. Optimal design of a novel cold spray gun nozzle at a limited space. J Therm Spray Technol 2005; 14: 391-396. 
19. Dykhuizen RC and Smith MF. Gas dynamic principles of cold spray. J Therm Spray Technol 1998; 7: 205-212.

20. Pandolfi M. Transonic swirling flow in axisymmetric nozzles. Meccanica 1976; 11: 157-161.

21. Liu Z, Ding J, Jiang W, et al. Numerical simulation of highly-swirling supersonic flow inside a Laval nozzle. Progr Comput Fluid Dyn Int J 2008; 8: 536-540.

22. Kitoh O. Experimental study of turbulent swirling flow in a straight pipe. J Fluid Mech 1991; 225: 445-479.

23. MacCormack RW. A numerical method for solving the equations of compressible viscous flow. AIAA J 1982; 20: 1275-1281.

24. Roe PL. Characteristic-based schemes for the Euler equations. Annu Rev Fluid Mech 1986; 18: 337-365.

25. Kafui KD, Thornton $\mathrm{C}$ and Adams MJ. Discrete particle-continuum fluid modelling of gas-solid fluidised beds. Chem Eng Sci 2002; 57: 2395-2410.

26. Wörner M. A compact introduction to the numerical modeling of multiphase flows. Forschungszentrum Karlsruhe, 2003.

27. Andrews MJ and O'rourke PJ. The multiphase particlein-cell (MP-PIC) method for dense particulate flows. Int J Multiphas Flow 1996; 22: 379-402.

28. Launder BE, Reece G Jr and Rodi W. Progress in the development of a Reynolds-stress turbulence closure. J Fluid Mech 1975; 68: 537-566.

29. Fedkiw RP. Coupling an Eulerian fluid calculation to a Lagrangian solid calculation with the ghost fluid method. J Comput Phys 2002; 175: 200-224.

30. FEPA. Fepa standard shapes and dimensions for precision superabrasives. FEPA SAFETY CODE, 2010.
31. Ferziger JH and Perić M. Computational methods for fluid dynamics. Vol. 3, Berlin: Springer, 2002.

32. Roache PJ. Perspective: a method for uniform reporting of grid refinement studies. J Fluid Eng 1994; 116: 405-413.

33. Salim SM and Cheah SC. Wall $\mathrm{y}^{+}$strategy for dealing with wall-bounded turbulent flows. In: Proceedings of the international multiconference of engineers and computer scientists, vol. 2, Hong Kong, China, 2009.

34. Stern F, Wilson RV, Coleman HW, et al. Comprehensive approach to verification and validation of CFD simulations, part 1: methodology and procedures. J Fluid Eng 2001; 123: 793-802.

35. Oberkampf WL and Trucano TG. Verification and validation in computational fluid dynamics. Progr Aero Sci 2002; 38: 209-272.

36. Marvin JG. Perspective on computational fluid dynamics validation. AIAA J 1995; 33: 1778-1787.

37. Xiao Q, Tsai H-M and Papamoschou D. Numerical investigation of supersonic nozzle flow separation. AIAA J 2007; 45: 532-541.

38. Abbasalizadeh M, Mirzaee I, Shirvani A, et al. An investigation into air-sand-water three-phase flow through the sandblasting nozzle. J Comput Multiphas Flow 2013; 5: 207-222.

39. Abbasalizadeh M. Investigation of three phase nozzle flow in an innovative sand blasting system. PhD Thesis, Anglia Ruskin University, Faculty of Science and Technology, Cambridge, UK, 2011. 